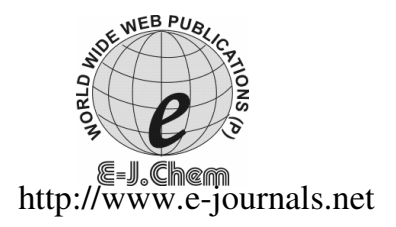

ISSN: 0973-4945; CODEN ECJHAO

E-Journal of Chemistry

2011, 8(2), 819-829

\title{
Non-Isothermal Decomposition of 2-(2-Hydroxybenzylideneamino)-3-phenylpropanoic Acid in Nitrogen Atmosphere
}

\author{
M. VENNILA, G. MANIKANDAN, \\ V. THANIKACHALAM and J. JAYABHARATHI* \\ Department of Chemistry, Annamalai University \\ Annamalai Nagar - 608 002, India \\ pvta1998@yahoo.co.in
}

Received 10 July 2010; Accepted 3 September 2010

\begin{abstract}
The non-isothermal decomposition properties of 2-(2-hydroxybenzylideneamino)-3-phenylpropanoic acid [HBAPPA] have been studied using microanalysis, FT-IR, UV, DTA, DTG and TG techniques. The TG studies were carried out at different heating rates of 10,15 and $20 \mathrm{~K} / \mathrm{min}$. The Schiff base decomposed in three stages. The kinetic parameters were deduced for each stage. A probable mechanism has been proposed for the decomposition process.
\end{abstract}

Keywords: Non-isothermal decomposition, Schiff base, Kinetic parameters.

\section{Introduction}

The well established thermogravimetric analysis (TGA), differential thermal analysis (DTA) and differential thermogravimetric analysis (DTG) techniques have been widely used for studying the thermal behaviour of various types of materials and the thermodynamic parameters for the decomposition processes could be evaluated. A method to determine the activation energy by using integral dynamic curves from TG at multiple heating rates has been proposed by Flynn-Wall ${ }^{1}$ and Ozawa ${ }^{2}$. Several studies have been carried out for the determination of kinetic parameters viz, activation energy, pre-exponential factor and life time of several materials such as polymers, organic and inorganic compounds ${ }^{3-6}$.

The Schiff base derived from salicylaldehyde and $L$-phenylalanine and its complexes are used as antibacterial ${ }^{7}$, antifungal ${ }^{7,8}$, antimalarial ${ }^{9}$ agents and also for DNA cleavage ${ }^{10}$, amino oxidase $^{11}$, deamination antiradical activity ${ }^{12}$, bio-distribution and blood clearance ${ }^{13}$. We are reporting here, the non-isothermal decomposition of 2-(2-hydroxybenzylideneamino)-3phenylpropanoic acid at different heating rates in nitrogen atmosphere. 


\section{Experimental}

Phenylalanine, salicylaldehyde and other chemicals used were of AnalaR grade from BDH.

\section{Preparation of 2-(2-hydroxybenzylideneamino)-3-phenylpropanoic acid}

The compound was synthesized by mixing an aqueous alcoholic solution of sodium salt of $L$-phenyalanine $(0.01 \mathrm{M})$ and salicylaldehyde $(0.01 \mathrm{M})$ (Scheme 1$)$. The reaction mixture was heated for about $4 \mathrm{~h}$. The resultant solution was cooled to room temperature and treated with 1:1 HCl. The pale yellow Schiff base was filtered, washed thoroughly with deionised water and dried in vacuum. The solid obtained was recrystallized using DMSO-water (50\%). The melting point of the compound is $189^{\circ} \mathrm{C}$ (lit: $189^{14}$ ). Micro anal calcd \% for $\mathrm{C}_{16} \mathrm{H}_{15} \mathrm{NO}_{3}$ : C, 71.37; H, 5.57; N, 5.20; found: C, 70.41; H, 5.34; N, 5.73. $\lambda_{\max }(\mathrm{DMF})=303 \mathrm{~nm}$, FT-IR $(\mathrm{KBr})$ : v $3071(\mathrm{C}-\mathrm{H}), 1624(\mathrm{C}=\mathrm{N}),(\mathrm{C}=\mathrm{O}) 1488(\mathrm{C}-\mathrm{N}), 1504(\mathrm{C}-\mathrm{O}), 1036(\mathrm{C}-\mathrm{O})$ and $747(\mathrm{C}-\mathrm{H}) \mathrm{cm}^{-1}$.

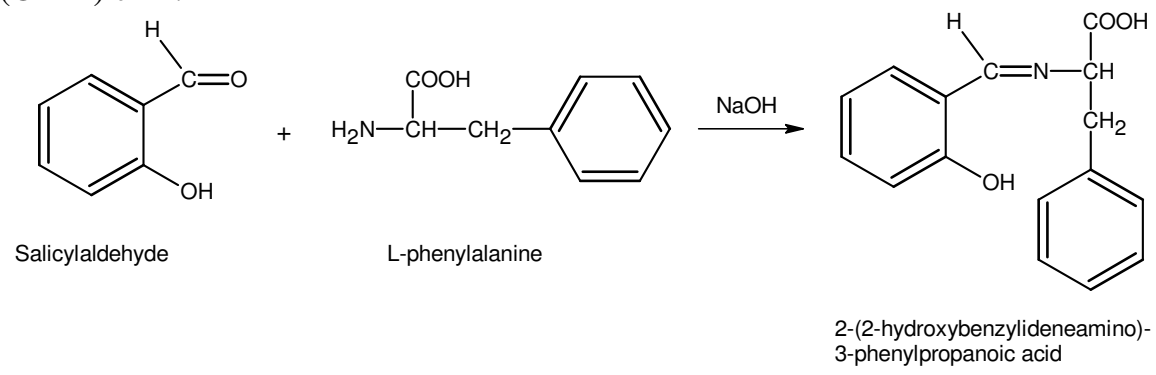

\section{Measurements}

\section{Scheme 1}

Microanalysis of the compound was carried out in a HERAEUS Carlo Erba 1108 model at Central Drug Research Institute, Lucknow, India. The UV-Visible spectrum of the sample was recorded on a HITACHIU $2001 \mathrm{UV}$-Visible spectrophotometer in DMF medium. The FT-IR spectrum was recorded on a AVATAR model 360 using $\mathrm{KBr}$ pellets. Thermogravimetric analysis was carried out using a NETZSCH - Gerate bare GMBH thermal analysis, STA 409 PC. The weight of the sample was maintained constant (10 mg) for all the heating rates.

\section{Kinetic theory}

The degree of conversion of the decomposition process is expressed as

$$
\alpha=\frac{\mathrm{m}_{0}-\mathrm{m}_{\mathrm{t}}}{\mathrm{m}_{0}-\mathrm{m}_{\infty}}
$$

Where, $\mathrm{m}_{0}$ is the initial mass of the sample, $\mathrm{m}_{\mathrm{t}}$ the mass of the sample at time $\mathrm{t}$ and $\mathrm{m}_{\infty}$, the final mass of the sample in that reaction. The general kinetic equation is written as

$$
\frac{\mathrm{d} \alpha}{\mathrm{dt}}=\mathrm{k}(\mathrm{T}) \mathrm{f}(\alpha)
$$

Where $\alpha$ is the degree of conversion of the sample and $t$ is time, $\mathrm{T}$ in the absolute temperature, $f(\alpha)$ is a function, the type of which depends on the reaction mechanism, $k(T)$, the temperature dependent rate constant, usually described by Arrhenius equation as,

$$
\mathrm{k}=\mathrm{A} \exp \left(-\frac{\mathrm{E}_{\mathrm{a}}}{\mathrm{RT}}\right)
$$


Where, $A$ is pre-exponential or frequency factor. $E_{a}$ is the activation energy and $R$ is the universal gas constant. Inserting Eqn (2) into Eqn (1) gives

$$
\frac{\mathrm{d} \alpha}{\mathrm{dt}}=\mathrm{A} \exp \left(-\frac{\mathrm{E}}{\mathrm{RT}}\right) \mathrm{f}(\alpha)
$$

An integration function is shown as

$$
g(\alpha)=\int_{0}^{\alpha} \frac{\mathrm{d} \alpha}{\mathrm{f}(\alpha)}=\frac{\mathrm{A}}{\beta} \int_{\mathrm{T}_{0}}^{\mathrm{T}} \exp \left(-\frac{\mathrm{E}}{\mathrm{RT}}\right) \mathrm{dT}
$$

Where $\mathrm{g}(\alpha)$ is the integral kinetic function or integral reaction model when its form is mathematically defined, $\beta=\mathrm{dT} / \mathrm{dt}$, the heating rate of decomposition. The kinetic parameters were calculated using model-fitting Coats and Redfern method ${ }^{15,16}$, model-free Flynn-WallOzawa $^{1,2}$ (FWO), Kissinger-Akahira and Sunose ${ }^{17,18}$ (KAS) and Tang ${ }^{19,20}$ (T) methods.

\section{Model-free method}

The following procedures are used for the determination of kinetic parameters by isoconversional methods.

\section{Flynn-Wall-Ozawa method ${ }^{1,2}$}

The isoconversional integral method suggested independently by Flynn- Wall ${ }^{1}$ and Ozawa ${ }^{2}$ uses Doyle's approximation of $\mathrm{p}(\mathrm{x})$. This method is based on the Eqn (6).

$$
\log \beta=\log \frac{\mathrm{AE}_{\mathrm{a}}}{\operatorname{Rg}(\alpha)}-2.315-0.4567 \frac{\mathrm{E}_{\mathrm{a}}}{\mathrm{RT}}
$$

Thus, for a constant $\alpha$ value, the plot $\log \beta$ versus (1/T), obtained from thermograms recorded at several heating rates should be a straight line whose slope can be used to evaluate the apparent activation energy.

\section{Kissinger-Akahira and Sunose method ${ }^{17,18}$}

This method is based on the Eqn (7).

$$
\ln \left[\frac{\beta}{\mathrm{T}^{2}}\right]=\ln \left[\frac{\mathrm{AR}}{\mathrm{E}_{\mathrm{a}} \mathrm{g}(\alpha)}\right]-\frac{\mathrm{E}_{\mathrm{a}}}{\mathrm{RT}}
$$

Thus, for a fixed $\alpha$ value, the plot of $\ln \left[\frac{\beta}{T^{2}}\right]$ versus $(1 / T)$ should be a straight line whose slope can be used to evaluate the apparent activation energy.

Tang method $^{19,20}$

This is based on the eqn. (8)

$$
\ln \left[\frac{\beta}{T^{1.894661}}\right]=\ln \left[\frac{\mathrm{AE}_{\mathrm{a}}}{\mathrm{g}(\alpha)}\right]-3.635041-1.894661 \ln \mathrm{E}_{\mathrm{a}}-1.001450 \frac{\mathrm{E}_{\mathrm{a}}}{\mathrm{RT}}
$$

Thus, for constant $\alpha$, the plot of $\ln \left[\frac{\beta}{T^{1.894661}}\right]$ versus $1 / T$, should be a straight line whose slope can be used to calculate $\mathrm{E}_{\mathrm{a}}$.

Model-fitting method (Coats and Refern) $)^{15,16}$

Modified Coats-Redfern equation is used for the determination of Arrhenius parameters. 


$$
\ln \left[\frac{\mathrm{g}(\alpha)}{\mathrm{T}^{2}}\right]=\ln \left[\frac{\mathrm{AR}}{\beta \mathrm{E}_{\mathrm{a}}}\left(1-\left[\frac{2 \mathrm{RT}}{\mathrm{E}_{\mathrm{a}}}\right]\right)\right]-\frac{\mathrm{E}_{\mathrm{a}}}{\mathrm{RT}}
$$

Different models are used for the calculation of kinetic parameters (Table 1).

Kissinger method ${ }^{17,21}$

Kissinger proposed a kinetic analysis method for thermal reactions. Models with different orders, $\left.[\mathrm{f}(\alpha)=1-\alpha)^{\mathrm{n}}\right]$ are based on taking the derivative of Eqn. (10) generating $\frac{\mathrm{d}^{2} \alpha}{\mathrm{dT}^{2}}$. According to Kissinger, the maximum reaction rate occurs when the second derivative is zero from which the following equation can be obtained:

$$
\frac{\mathrm{E}_{\mathrm{a}} \beta}{\mathrm{RT}_{\mathrm{m}}^{2}}=\mathrm{A}\left(\mathrm{n}(1-\alpha)_{\mathrm{m}}^{\mathrm{n}-1}\right) \mathrm{e} \frac{\mathrm{E}_{\mathrm{a}}}{\mathrm{RT}_{\mathrm{m}}}
$$

Where, $T_{m}$ is the temperature of the maximum rate and $\alpha_{m}$ is the conversion value at that rate. The maximum reaction rate represents the peak (i.e. inflection point) of a DSC or DTG curve. Taking the natural logarithm of Eqn. (10) and rearranging gives,

$$
\ln \left(\frac{\beta}{T_{m}^{2}}\right)=\ln \left(\frac{\operatorname{AR}\left(n(1-\alpha)_{m}^{n-1}\right)}{E_{a}}\right)-\frac{E_{a}}{R_{m}}
$$

The activation energy is obtained by plotting the left-hand side of the above equation versus $\frac{1}{T_{m}}$ for a series of runs at different heating rates. Eqn. (11) has been generalized to any reaction model $(f(\alpha))$. The peak temperatures are noted from DTG curves at different heating rates $^{22,23}$. The thermodynamic parameters can be calculated using the following equations:

$$
\begin{aligned}
\mathrm{A} \exp (-\mathrm{E} / \mathrm{RT}) & =v \exp \left(-\Delta \mathrm{G}^{\neq} / \mathrm{RT}\right) \\
\Delta \mathrm{H}^{\neq} & =\mathrm{E}-\mathrm{RT} \\
\Delta \mathrm{G}^{\neq} & =\Delta \mathrm{H}^{\neq}-\mathrm{T} \Delta \mathrm{S}^{\neq}
\end{aligned}
$$

Where $\Delta \mathrm{G}^{\neq}$is the Gibbs free energy of activation, $\Delta \mathrm{H}^{\neq}$the enthalpy of activation, $\Delta \mathrm{S}^{\neq}$the entropy of activation and $v$ the Einstein vibrational frequency, $v=k_{B} T / h$ (where $k_{B}$ and $h$ are Boltzmann and Planck's constants, respectively). The values of entropy, enthalpy and free energy of activation at the peak temperatures are obtained on the basis of Eqns. (12) - (14).

\section{Results and Discussion}

TG, DTA and DTG curves of salicylidene-phenylalanine are shown in Figure 1 at various heating rates, i.e. 10,15 and $20 \mathrm{~K} / \mathrm{min}$. As can be seen from Figure 1, the compound is stable up to $200{ }^{\circ} \mathrm{C}$ in all heating rates. After the endothermic process of melting at $104.8{ }^{\circ} \mathrm{C}$ at the heating rate of $10 \mathrm{~K} / \mathrm{min}$, it shows three decomposition stages of mass loss. The first stage starts at $480 \mathrm{~K}$ and ends at about $550 \mathrm{~K}$ with the corresponding mass loss of 68.71 , 62.27 and $65.90 \%$ at different heating rates. It is probably due to the partial release of phenylalanine (found 62.27, calculated $60.57 \%$ ) characterized by a strong endothermic peak. The second stage starts at $550 \mathrm{~K}$ and ends at $693 \mathrm{~K}$, with the mass loss of 16.48, 16.78 and $22.48 \%$ respectively for different heating rates (weak endothermic) attributed to the partial decomposition of salicylaldehyde (cyclobutadione) (found 16.46; calculated 19.33\%) with weak endothermic peak observed at $387.3{ }^{\circ} \mathrm{C}$. In the third stage, which starts at $694 \mathrm{~K}$ ends at $1073 \mathrm{~K}$. This is due to the mass loss of cyclopropanic oxide. Similar trends are observed for other heating rates also. 

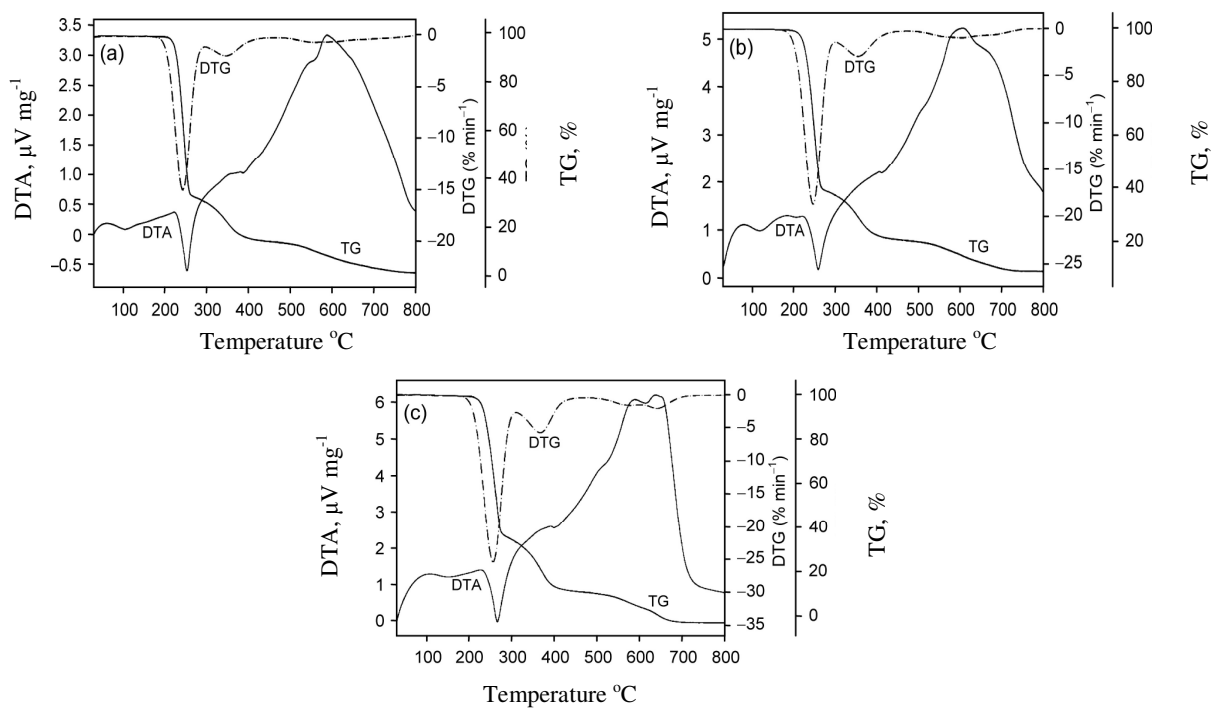

Figure 1. TG, DTA and DTG curves of HBAPPA at a) $10 \mathrm{~K} \mathrm{~min}^{-1}$; b) $15 \mathrm{~K} \mathrm{~min}^{-1}$ and c) $20 \mathrm{~K} \mathrm{~min}^{-1}$ heating rates in nitrogen atmosphere.

\section{Kinetic analysis}

TG, DTG, DTA curves of the title compound are shown in Figure 1. The data obtained for the non-isothermal decomposition process at 10,15 and $20 \mathrm{~K} / \mathrm{min}$ were processed according to equation (1). The variation of $E_{a}$ with $\alpha$ for all the three decomposition stages are given in Figures. 2-4. It is seen that $E_{a}$ value depends upon the extent of conversion in stages I and III (Figures $2 \& 4$ ), whereas in stage II (Figure 3) it is independent of the extent of conversion $(0.2 \leq \alpha \leq 0.8)$. The data indicate that the kinetics are likely to be governed by a single step reaction i.e., $\mathrm{F}_{1}$ mechanism for state II, whereas as multi-step reactions are involved in stages I and III. It is seen from the Figures 2 and 4, that there is a decrease in activation energy for stage I and for the stage III it increases up to $\alpha \leq 0.5$ and then decreases. This suggests that it follows a multi-step kinetics. Conversely, in stage II, the $\mathrm{E}_{\mathrm{a}}$ values do not significantly vary with $\alpha(0.1 \leq \alpha \leq 0.8)$. Figure 2 shows a decrease in activation energy from 200 to $102 \mathrm{~kJ} / \mathrm{mol}$ as $\alpha$ increases. In second stage, the $\mathrm{E}_{\mathrm{a}}$ value increases from 93 to $190 \mathrm{~kJ} / \mathrm{mol}$ (Figure 3). In the third stage, the $E_{a}$ value increases from 26 to $96 \mathrm{~kJ} / \mathrm{mol}(0.1 \leq \alpha \leq 0.5)$ (Figure 4).

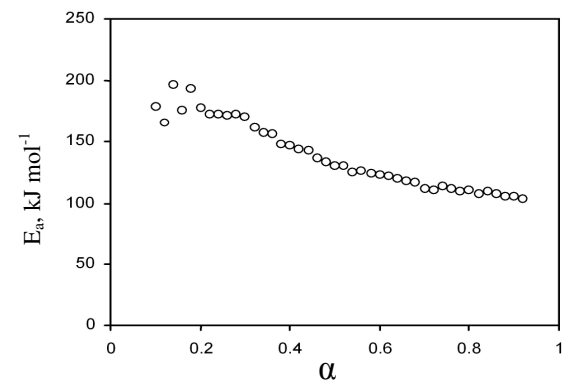

Figure 2. Variation of the $\mathrm{E}_{\mathrm{a}}$ with $\alpha$ for the first stage decomposition of HBAPPA by FWO method

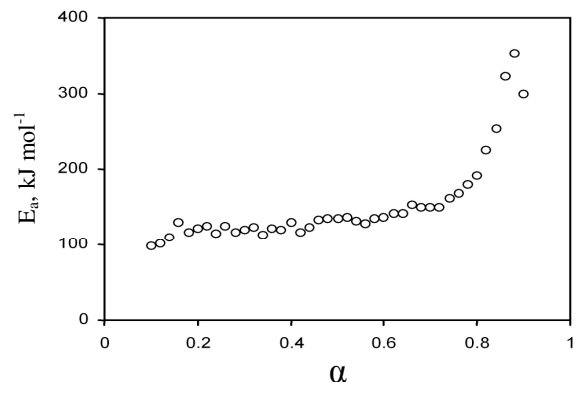

Figure 3. Variation of $E_{a}$ with $\alpha$ for the second stage decomposition of HBAPPA by FWO method 


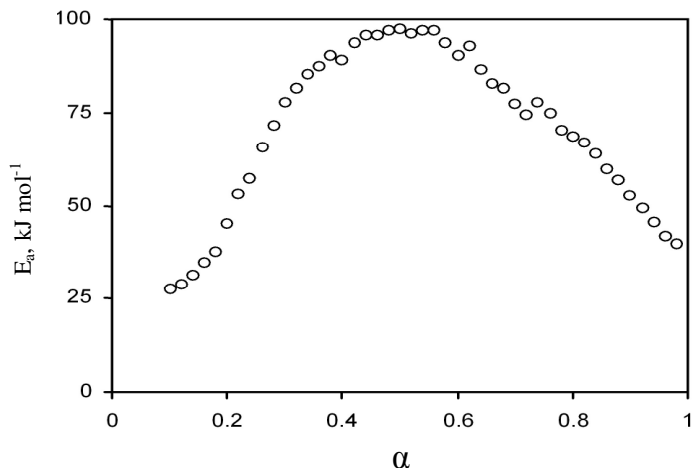

Figure 4. Variation of $E_{a}$ with $\alpha$ for the third stage decomposition of HBAPPA by FWO method

\section{Invariant kinetic parameters}

The invariant kinetic parameters, $\mathrm{E}_{\mathrm{inv}}$ and $\mathrm{A}_{\mathrm{inv}}$ are calculated using Coats-Redfern method $^{15,16,24}$ (Table 1). The values of $E_{a}$ and $\ln A$ depend on the kinetic model as well as on the heating rate, as shown in Tables 2-4. The evaluation of the invariant parameters is performed using supper correlation equation and the values are listed in Table 5.

$$
a_{\beta}=\ln A_{i n v}-b_{\beta} E_{\text {inv }}
$$

Table 1. Algebraic expressions of $f(\alpha)$ and $g(\alpha)$ for the reaction models considered in the present work ${ }^{15,16}$

\begin{tabular}{cccc}
\hline Symbol & Reaction Model & $\begin{array}{c}\text { Differential form } \\
f(\alpha)=\frac{1}{k} \frac{\mathrm{d} \alpha}{\mathrm{d} t}\end{array}$ & $\begin{array}{c}\text { Integral form }^{a} g(\alpha) \\
=k t\end{array}$ \\
\hline P2 & Power law & $3 \alpha^{(2 / 3)}$ & $\begin{array}{c}\alpha^{(1 / 3)} \\
\alpha^{(1 / 2)}\end{array}$ \\
P3 & Power law & $2 \alpha^{(1 / 2)}$ & $\alpha^{2}$ \\
D1 & 1-D Diffusion & $1 / 2 \alpha$ & $\alpha^{(3 / 2)}$ \\
P4 & Power law & $2 / 3 \alpha^{(-1 / 2)}$ & $-\ln (1-\alpha)$ \\
F1 & First-order (mampel) & $(1-\alpha)$ & {$[-\ln (1-\alpha)]^{1 / 4}$} \\
A4 & Avarami-Erofe'ev (n=4) & $4(1-\alpha)[-\ln (1-\alpha)]^{3 / 4}$ & {$[-\ln (1-\alpha)]^{1 / 3}$} \\
A3 & Avarami-Erofe'ev (n=3) & $3(1-\alpha)[-\ln (1-\alpha)]^{2 / 3}$ & {$[-\ln (1-\alpha)]^{1 / 2}$} \\
A2 & Avarami-Erofe'ev (n=2) & $2(1-\alpha)[-\ln (1-\alpha)]^{1 / 2}$ & {$\left[1-(1-\alpha)^{1 / 3}\right]^{2}$} \\
D3 & 3-D Diffusion-Jander Eqn. & $3 / 2(1-\alpha)^{2 / 3} / 2\left[1-(1-\alpha)^{1 / 3}\right]^{-1}$ & {$\left[1-(1-\alpha)^{1 / 3}\right]$} \\
R3 & Contracting area & $3(1-\alpha)^{2 / 3}$ & {$\left[1-(1-\alpha)^{1 / 2}\right]$} \\
R2 & Contracting area & $2(1-\alpha)^{1 / 2}$ & $(1-\alpha)^{-1}-1$ \\
F2 & Second-order & $(1-\alpha)^{2}$ & $(-\ln (1-\alpha))^{-1}$ \\
D2 & 2-D Diffusion & $(1-\alpha) \ln (1-\alpha)^{+2}$ & $3 / 2\left((1-\alpha)^{-1 / 3}-1\right)^{-1}$ \\
D4 & Ginstling-Brounshtein & $(1-2 \alpha / 3)-(1-\alpha)^{2 / 3}$ &
\end{tabular}

${ }^{a}$ In some references $f(\alpha)$ and $g(\alpha)$ have opposite designations 
Table 2. Kinetic parameters for the first stage decomposition of HBAPPA

\begin{tabular}{ccccccccccc}
\hline \multirow{2}{*}{$\begin{array}{c}\text { Kinetic } \\
\text { model }\end{array}$} & \multicolumn{3}{c}{$\beta=10, \mathrm{~K} \mathrm{~min}^{-1}$} & \multicolumn{3}{c}{$\beta=15, \mathrm{~K} \mathrm{~min}^{-1}$} & \multicolumn{3}{c}{$\beta=20, \mathrm{~K} \mathrm{~min}^{-1}$} \\
& $\begin{array}{c}E_{\mathrm{a}} \\
\mathrm{kJ} \mathrm{mol}^{-1}\end{array}$ & $\begin{array}{c}\ln A, \\
\mathrm{~s}^{-1}\end{array}$ & $r$ & $\begin{array}{c}E_{\mathrm{a}} \\
\mathrm{kJ} \mathrm{mol}^{-1}\end{array}$ & $\begin{array}{c}\ln A, \\
\mathrm{~s}^{-1}\end{array}$ & $r$ & $\begin{array}{c}E_{\mathrm{a}} \\
\mathrm{kJ} \mathrm{mol}^{-1}\end{array}$ & $\begin{array}{c}\ln A, \\
\mathrm{~s}^{-1}\end{array}$ & $r$ \\
\hline P2 & 68.71 & 14.50 & -0.990 & 60.14 & 12.60 & -0.987 & 53.46 & 11.08 & -0.983 \\
$\mathrm{P} 3$ & 42.92 & 8.13 & -0.988 & 37.18 & 6.94 & -0.984 & 32.70 & 5.96 & -0.980 \\
P4 & 30.09 & 4.85 & -0.986 & 25.76 & 4.00 & -0.982 & 22.38 & 3.29 & -0.976 \\
$\mathrm{~F} 1$ & 209.78 & 48.61 & -0.999 & 186.11 & 42.88 & -0.999 & 167.61 & 38.35 & -0.999 \\
$\mathrm{~F} 2$ & 298.86 & 70.30 & -0.987 & 265.97 & 62.22 & -0.988 & 240.19 & 55.83 & -0.989 \\
$\mathrm{~F} 3$ & 409.34 & 97.03 & -0.968 & 365.04 & 86.04 & -0.970 & 330.24 & 77.36 & -0.971 \\
$\mathrm{D} 1$ & 315.62 & 79.96 & -0.992 & 280.80 & 71.41 & -0.990 & 253.73 & 64.72 & -0.988 \\
$\mathrm{D} 2$ & 335.44 & 76.81 & -0.996 & 297.79 & 67.53 & -0.995 & 268.46 & 60.26 & -0.993 \\
$\mathrm{D} 3$ & 380.20 & 86.16 & -0.999 & 337.92 & 75.70 & -0.999 & 304.93 & 67.49 & -0.997 \\
$\mathrm{D} 4$ & 374.89 & 84.62 & -0.997 & 332.87 & 74.24 & -0.995 & 300.16 & 66.11 & -0.993 \\
$\mathrm{~A} 2$ & 100.63 & 22.55 & -0.999 & 88.75 & 19.81 & -0.999 & 79.45 & 17.62 & -0.998 \\
$\mathrm{~A} 3$ & 63.61 & 13.50 & -0.999 & 55.72 & 11.77 & -0.999 & 49.55 & 10.37 & -0.998 \\
$\mathrm{~A} 4$ & 46.05 & 9.11 & -0.999 & 40.07 & 7.85 & -0.999 & 35.38 & 6.82 & -0.998 \\
$\mathrm{R} 2$ & 174.78 & 39.34 & -0.998 & 154.73 & 34.53 & -0.997 & 139.10 & 30.74 & -0.995 \\
$\mathrm{R} 3$ & 164.91 & 37.19 & -0.996 & 145.89 & 32.64 & -0.995 & 131.06 & 29.05 & -0.993 \\
\hline
\end{tabular}

Table 3. Kinetic parameters for the second stage decomposition of HBAPPA

\begin{tabular}{ccccccccccc}
\hline \multirow{2}{*}{$\begin{array}{c}\text { Kinetic } \\
\text { model }\end{array}$} & \multicolumn{3}{c}{$\beta=10, \mathrm{~K} \mathrm{~min}^{-1}$} & \multicolumn{3}{c}{$\beta=15, \mathrm{~K} \mathrm{~min}^{-1}$} & \multicolumn{3}{c}{$\beta=20, \mathrm{~K} \mathrm{~min}^{-1}$} \\
& $\begin{array}{c}E_{\mathrm{a}} \\
\mathrm{kJ} \mathrm{mol}^{-1}\end{array}$ & $\begin{array}{c}\ln A, \\
\mathrm{~s}^{-1}\end{array}$ & $r$ & $\begin{array}{c}E_{\mathrm{a}} \\
\mathrm{kJ} \mathrm{mol}^{-1}\end{array}$ & $\begin{array}{c}\ln A, \\
\mathrm{~s}^{-1}\end{array}$ & $r$ & $\begin{array}{c}E_{\mathrm{a}} \\
\mathrm{kJ} \mathrm{mol}^{-1}\end{array}$ & $\begin{array}{c}\ln A, \\
\mathrm{~s}^{-1}\end{array}$ & $r$ \\
\hline P2 & 23.89 & 1.76 & -0.985 & 24.27 & 2.21 & -0.983 & 27.56 & 3.16 & -0.995 \\
P3 & 12.57 & -0.98 & -0.976 & 12.80 & -0.55 & -0.973 & 14.94 & 0.24 & -0.992 \\
P4 & 6.94 & -2.62 & -0.954 & 7.10 & -2.19 & -0.950 & 8.67 & -1.5 & -0.985 \\
F1 & 84.32 & 14.96 & -1.000 & 85.60 & 15.50 & -0.999 & 94.16 & 17.26 & -0.996 \\
F2 & 120.62 & 22.98 & -0.988 & 122.52 & 23.59 & -0.989 & 133.35 & 25.66 & -0.977 \\
F3 & 165.35 & 32.70 & -0.970 & 168.04 & 33.40 & -0.971 & 181.51 & 35.85 & -0.954 \\
D1 & 137.48 & 32.44 & -0.993 & 139.35 & 33.04 & -0.992 & 153.34 & 35.71 & -0.997 \\
D2 & 140.32 & 24.89 & -0.997 & 142.32 & 25.49 & -0.996 & 156.97 & 28.26 & -0.999 \\
D3 & 158.88 & 27.43 & -0.999 & 161.19 & 28.06 & -0.999 & 177.11 & 31.02 & -0.999 \\
D4 & 157.23 & 26.82 & -0.996 & 159.52 & 27.46 & -0.995 & 175.81 & 30.49 & -0.999 \\
A2 & 37.16 & 5.02 & -0.999 & 37.76 & 5.50 & -0.999 & 41.98 & 6.57 & -0.995 \\
A3 & 21.16 & 1.37 & -0.999 & 21.54 & 1.82 & -0.999 & 24.28 & 2.67 & -0.994 \\
A4 & 13.58 & -0.54 & -0.999 & 13.84 & -0.10 & -0.999 & 15.88 & 0.65 & -0.993 \\
R2 & 69.85 & 11.01 & -0.998 & 70.89 & 11.53 & -0.997 & 78.48 & 13.14 & -0.999 \\
R3 & 65.74 & 10.35 & -0.996 & 66.71 & 10.86 & -0.995 & 74.00 & 12.44 & -0.999 \\
\hline
\end{tabular}

Table 4. Kinetic parameters for the third stage decomposition of HBAPPA

\begin{tabular}{cccccccccc}
\hline \multirow{2}{*}{$\begin{array}{c}\text { Kinetic } \\
\text { model }\end{array}$} & \multicolumn{3}{c}{$\beta=10\left(\mathrm{~K} \mathrm{~min}^{-1}\right)$} & \multicolumn{3}{c}{$\beta=15, \mathrm{~K} \mathrm{~min}^{-1}$} & \multicolumn{3}{c}{$\beta=20, \mathrm{~K} \mathrm{~min}^{-1}$} \\
& $\begin{array}{c}E_{\mathrm{a}} \\
\mathrm{kJ} \mathrm{mol}^{-1}\end{array}$ & $\begin{array}{c}\ln A, \\
\mathrm{~s}^{-1}\end{array}$ & $r$ & $\begin{array}{c}E_{\mathrm{a}} \\
\mathrm{kJ} \mathrm{mol}^{-1}\end{array}$ & $\begin{array}{c}\ln A, \\
\mathrm{~s}^{-1}\end{array}$ & $r$ & $\begin{array}{c}E_{\mathrm{a}} \\
\mathrm{kJ} \mathrm{mol}^{-1}\end{array}$ & $\begin{array}{c}\ln A, \\
\mathrm{~s}^{-1}\end{array}$ & $r$ \\
\hline $\mathrm{P} 2$ & 14.69 & -1.95 & -0.988 & 16.18 & -1.42 & -0.925 & 20.67 & -0.39 & -0.827 \\
$\mathrm{P} 3$ & 5.31 & -4.19 & -0.963 & 5.97 & -3.70 & -0.793 & 8.77 & -2.72 & -0.678 \\
$\mathrm{P} 4$ & 0.65 & -6.89 & -0.521 & 0.90 & -6.22 & -0.245 & 2.85 & -4.57 & -0.366 \\
\hline
\end{tabular}




\begin{tabular}{cccccccccc}
\hline F1 & 64.14 & 6.68 & -0.973 & 72.80 & 7.91 & -0.995 & 89.46 & 10.35 & -0.966 \\
F2 & 93.55 & 11.81 & -0.937 & 109.33 & 13.87 & -0.991 & 136.82 & 17.66 & -0.994 \\
F3 & 129.82 & 17.96 & -0.903 & 154.71 & 21.08 & -0.976 & 196.08 & 26.60 & -0.998 \\
D1 & 113.04 & 21.33 & -0.996 & 123.06 & 22.47 & -0.979 & 143.85 & 25.29 & -0.932 \\
D2 & 110.75 & 12.30 & -0.992 & 122.10 & 13.59 & -0.984 & 145.50 & 16.73 & -0.938 \\
D3 & 125.69 & 13.35 & -0.985 & 140.39 & 15.01 & -0.992 & 168.84 & 18.79 & -0.957 \\
D4 & 124.82 & 12.94 & -0.991 & 137.61 & 14.33 & -0.984 & 164.22 & 17.86 & -0.941 \\
A2 & 25.38 & 0.34 & -0.961 & 29.21 & 1.17 & -0.992 & 37.24 & 2.63 & -0.951 \\
A3 & 12.24 & -2.22 & -0.935 & 14.43 & -1.51 & -0.983 & 19.52 & -0.33 & -0.922 \\
A4 & 6.00 & -3.80 & -0.868 & 7.42 & -3.11 & -0.963 & 11.13 & -1.99 & -0.871 \\
R2 & 52.47 & 3.88 & -0.987 & 58.49 & 4.81 & -0.985 & 71.16 & 6.75 & -0.937 \\
R3 & 49.15 & 3.55 & -0.990 & 54.46 & 4.39 & -0.980 & 66.05 & 6.20 & -0.926 \\
\hline
\end{tabular}

Table 5. Compensation effect parameters for several combinations of kinetic models for the decomposition of HBAPPA

\begin{tabular}{|c|c|c|c|c|c|c|}
\hline \multicolumn{7}{|c|}{ Stage I } \\
\hline \multirow{2}{*}{$\begin{array}{c}\beta \\
\mathrm{K} \min ^{-1}\end{array}$} & \multicolumn{3}{|c|}{ AKM } & \multicolumn{3}{|c|}{ AKM-\{D1; D3; D4 $\}$} \\
\hline & $\mathrm{a}_{\beta}, \mathrm{A} / \mathrm{s}^{-1}$ & $\mathrm{~b}_{\beta} / \mathrm{mol} \mathrm{J}^{-1}$ & $r$ & $\mathrm{a}_{\beta}, \mathrm{A} / \mathrm{s}^{-1}$ & $\mathrm{~b}_{\beta} / \mathrm{mol} \mathrm{J}^{-1}$ & $r$ \\
\hline 10 & -1.78774 & 0.23857 & 0.997 & -2.07343 & 0.23999 & 0.999 \\
\hline 15 & -1.54495 & 0.23678 & 0.997 & -1.82566 & 0.23885 & 0.999 \\
\hline 20 & -1.40395 & 0.23506 & 0.996 & -1.67965 & 0.23676 & 0.999 \\
\hline$\beta$ & \multicolumn{3}{|c|}{ AKM-\{F2; F3; D1; D2; D3; D4 $\}$} & \multicolumn{3}{|c|}{$\mathrm{AKM}-\{\mathrm{D} 1 ; \mathrm{D} 2 ; \mathrm{D} 3 ; \mathrm{D} 4\}$} \\
\hline $\mathrm{K} \min ^{-1}$ & $\mathrm{a}_{\beta}, \mathrm{A} / \mathrm{s}^{-1}$ & $\mathrm{~b}_{\beta} / \mathrm{mol} \mathrm{J}^{-1}$ & $R$ & $\mathrm{a}_{\beta}, \mathrm{A} / \mathrm{s}^{-1}$ & $\mathrm{~b}_{\beta} / \mathrm{mol} \mathrm{J}^{-1}$ & $r$ \\
\hline 10 & -2.01378 & 0.2395 & 0.999 & -2.23121 & 0.24207 & 0.999 \\
\hline 15 & -1.77254 & 0.2379 & 0.999 & -1.97946 & 0.24068 & 0.999 \\
\hline 20 & -1.63184 & 0.23634 & 0.999 & -1.82942 & 0.23932 & 0.999 \\
\hline \multicolumn{7}{|c|}{ Stage II } \\
\hline \multirow{2}{*}{$\begin{array}{c}\beta, \\
\mathrm{K} \min ^{-1}\end{array}$} & \multicolumn{3}{|c|}{$\mathrm{AKM}$} & \multicolumn{3}{|c|}{$\mathrm{AKM}-\{\mathrm{D} 1 ; \mathrm{D} 3 ; \mathrm{D} 4\}$} \\
\hline & $\mathrm{a}_{\beta}, \mathrm{A} / \mathrm{s}^{-1}$ & $\mathrm{~b}_{\beta} / \mathrm{mol} \mathrm{J}^{-1}$ & $r$ & $\mathrm{a}_{\beta}, \mathrm{A} / \mathrm{s}^{-1}$ & $\mathrm{~b}_{\beta} / \mathrm{mol} \mathrm{J}^{-1}$ & $r$ \\
\hline 10 & -3.30911 & 0.21189 & 0.985 & -3.51601 & 0.21417 & 0.998 \\
\hline 15 & -2.90037 & 0.21008 & 0.985 & -3.10825 & 0.21252 & 0.998 \\
\hline 20 & -2.50933 & 0.20563 & 0.987 & -2.72024 & 0.20785 & 0.998 \\
\hline \multirow{2}{*}{$\begin{array}{c}\beta, \\
\mathrm{K} \min ^{-1}\end{array}$} & \multicolumn{3}{|c|}{$\mathrm{AKM}-\{\mathrm{F} 3 ; \mathrm{D} 1 ; \mathrm{D} 2 ; \mathrm{D} 3 ; \mathrm{D} 4\}$} & \multicolumn{3}{|c|}{$\begin{array}{c}\mathrm{AKM}-\{\mathrm{P} 2 ; \mathrm{P} 3 ; \mathrm{P} 4 ; \mathrm{D} 1 ; \mathrm{D} 2 ; \mathrm{D} 3 ; \\
\mathrm{D} 4 ; \mathrm{A} 2 ; \mathrm{A} 3 ; \mathrm{A} 4\}\end{array}$} \\
\hline & $\mathrm{a}_{\beta}, \mathrm{A} / \mathrm{s}^{-1}$ & $\mathrm{~b}_{\beta} / \mathrm{mol} \mathrm{J}^{-1}$ & $r$ & $\mathrm{a}_{\beta}, \mathrm{A} / \mathrm{s}^{-1}$ & $\mathrm{~b}_{\beta} / \mathrm{mol} \mathrm{J}^{-1}$ & $r$ \\
\hline 10 & -3.64362 & 0.21882 & 0.998 & -4.40125 & 0.22536 & 0.999 \\
\hline 15 & -3.2368 & 0.21713 & 0.998 & -3.97938 & 0.22341 & 0.999 \\
\hline 20 & -2.85093 & 0.21196 & 0.999 & -3.68962 & 0.2187 & 0.999 \\
\hline$\beta$, & \multicolumn{3}{|c|}{$\overline{\mathrm{AKM}}-\{\mathrm{P} 2 ; \mathrm{A} 2\}$} & & & \\
\hline $\mathrm{K} \min ^{-1}$ & $\mathrm{a}_{\beta}, \mathrm{A} / \mathrm{s}^{-1}$ & $\mathrm{~b}_{\beta} / \mathrm{mol} \mathrm{J}^{-1}$ & $r$ & & & \\
\hline 10 & -3.13797 & 0.20397 & 0.999 & & & \\
\hline 15 & -2.73394 & 0.2026 & 0.999 & & & \\
\hline 20 & -2.33566 & 0.19852 & 0.999 & & & \\
\hline
\end{tabular}




\begin{tabular}{|c|c|c|c|c|c|c|}
\hline \multicolumn{7}{|c|}{ Stage III } \\
\hline \multirow{2}{*}{$\begin{array}{c}\beta \\
\mathrm{K} \min ^{-1}\end{array}$} & \multicolumn{3}{|c|}{ AKM } & \multicolumn{3}{|c|}{$\begin{array}{c}\text { AKM - }\{\text { P2; P3; P4; D1; D2; D3; } \\
\text { D4; A2; A3; A4; R2; R3 }\end{array}$} \\
\hline & $\mathrm{a}_{\beta}, \mathrm{A} / \mathrm{s}^{-1}$ & $\mathrm{~b}_{\beta} / \mathrm{mol} \mathrm{J}^{-1}$ & $r$ & $\mathrm{a}_{\beta}, \mathrm{A} / \mathrm{s}^{-1}$ & $\mathrm{~b}_{\beta} / \mathrm{mol} \mathrm{J}^{-1}$ & $r$ \\
\hline 10 & -4.83949 & 0.16998 & 0.962 & -4.30115 & 0.17166 & 0.999 \\
\hline 15 & -4.34544 & 0.16031 & 0.966 & -3.75791 & 0.16071 & 0.999 \\
\hline 20 & -3.65528 & 0.15106 & 0.974 & -3.24571 & 0.15235 & 0.999 \\
\hline \multirow{2}{*}{$\begin{array}{c}\beta \\
\mathrm{K} \min ^{-1}\end{array}$} & \multicolumn{3}{|c|}{$\begin{array}{c}\mathrm{AKM}-\{\mathrm{P} 3 ; \mathrm{P} 4 ; \mathrm{D} 1 ; \mathrm{D} 2 ; \mathrm{D} 3 ; \mathrm{D} 4 ; \\
\mathrm{A} 2 ; \mathrm{R} 2\}\end{array}$} & \multicolumn{3}{|c|}{$\begin{array}{c}\mathrm{AKM}-\{\mathrm{P} 2 ; \mathrm{P} 3 ; \mathrm{P} 4 ; \mathrm{D} 1 ; \mathrm{D} 2 ; \mathrm{D} 3 ; \\
\mathrm{D} 4 ; \mathrm{A} 2 ; \mathrm{A} 3 ; \mathrm{A} 4 ; \mathrm{R} 2\}\end{array}$} \\
\hline & $\mathrm{a}_{\beta}, \mathrm{A} / \mathrm{s}^{-1}$ & $\mathrm{~b}_{\beta} / \mathrm{mol} \mathrm{J}^{-1}$ & $r$ & $\mathrm{a}_{\beta}, \mathrm{A} / \mathrm{s}^{-1}$ & $\mathrm{~b}_{\beta} / \mathrm{mol} \mathrm{J}^{-1}$ & $r$ \\
\hline 10 & -4.62622 & 0.17428 & 0.999 & -4.90202 & 0.17706 & 0.999 \\
\hline 15 & -4.13248 & 0.16336 & 0.999 & -4.34541 & 0.16517 & 0.999 \\
\hline 20 & -3.59587 & 0.15428 & 0.999 & -3.82654 & 0.15584 & 0.999 \\
\hline \multirow{2}{*}{$\begin{array}{c}\beta, \\
\mathrm{K} \mathrm{min}^{-1}\end{array}$} & \multicolumn{3}{|c|}{$\begin{array}{c}\mathrm{AKM}-\{\mathrm{P} 2 ; \mathrm{P} 3 ; \mathrm{P} 4 ; \mathrm{D} 1 ; \mathrm{D} 2 ; \mathrm{D} 3 ; \\
\mathrm{D} 4 ; \mathrm{A} 3 ; \mathrm{A} 4\}\end{array}$} & \multicolumn{3}{|c|}{ AKM - \{P4; D1; D2; D3; D4 $\}$} \\
\hline & $\mathrm{a}_{\beta}, \mathrm{A} / \mathrm{s}^{-1}$ & $\mathrm{~b}_{\beta} / \mathrm{mol} \mathrm{J}^{-1}$ & $r$ & $\mathrm{a}_{\beta}, \mathrm{A} / \mathrm{s}^{-1}$ & $\mathrm{~b}_{\beta} / \mathrm{mol} \mathrm{J}^{-1}$ & $r$ \\
\hline 10 & -4.61892 & 0.17354 & 0.999 & -4.69687 & 0.17455 & 0.998 \\
\hline 15 & -4.12506 & 0.1628 & 0.998 & -4.21698 & 0.16379 & 0.998 \\
\hline 20 & -3.64697 & 0.15427 & 0.998 & -3.67827 & 0.15458 & 0.999 \\
\hline
\end{tabular}

The plot of $a_{\beta}$ versus $b_{\beta}$, obtained for three constant heating rates, is a straight line whose parameters allow the computation of $\ln \mathrm{A}_{\text {inv }}$ and $\mathrm{E}_{\text {inv }}$ (Table 6). For several groups of apparent activation parameters listed in Table 5, obtained by different kinetic models, we tried to establish the best correlation and the closest value to the mean isoconversional activation energies [FWO; $\left.E_{a}=138.97 \pm 4.31 \mathrm{~kJ} / \mathrm{mol}\right]$. For $A K M-\left\{D_{1}-D_{4}\right)$, the plot of $\ln A$ versus $\mathrm{E}_{\mathrm{a}}$ has the highest correlation coefficient $\mathrm{E}_{\mathrm{a}}=146.23 \mathrm{~kJ} / \mathrm{mol} ; \ln \mathrm{A}=33.18$; $(\mathrm{r}=-0.990)$ and is a straight line for stage $\mathrm{I}$.

Table 6. IKP for several combinations of kinetic models for HBAPPA

\begin{tabular}{|c|c|c|c|}
\hline Kinetic model & $\begin{array}{c}E_{\text {inv }} \\
\mathrm{kJ} \mathrm{mol}^{-1}\end{array}$ & $\ln A_{\text {inv }}$ & $r$ \\
\hline \multicolumn{4}{|l|}{ Stage I } \\
\hline AKM & 109.52 & 24.35 & -0.990 \\
\hline $\mathrm{AKM}-\{\mathrm{D} 1 ; \mathrm{D} 3 ; \mathrm{D} 4\}$ & 122.06 & 27.23 & -0.990 \\
\hline $\mathrm{AKM}-\{\mathrm{F} 2 ; \mathrm{F} 3 ; \mathrm{D} 1 ; \mathrm{D} 2 ; \mathrm{D} 3 ; \mathrm{D} 4\}$ & 120.99 & 26.98 & -0.989 \\
\hline $\mathrm{AKM}-\{\mathrm{D} 1 ; \mathrm{D} 2 ; \mathrm{D} 3 ; \mathrm{D} 4\}$ & 146.23 & 33.18 & -0.990 \\
\hline $\mathrm{AKM}-\{\mathrm{F} 1 ; \mathrm{F} 2 ; \mathrm{F} 3 ; \mathrm{D} 1 ; \mathrm{D} 2 ; \mathrm{D} 3 ; \mathrm{D} 4\}$ & 104.13 & 22.85 & -0.990 \\
\hline \multicolumn{4}{|l|}{ Stage II } \\
\hline AKM & 128.76 & 23.99 & -0.935 \\
\hline $\mathrm{AKM}-\{\mathrm{D} 1 ; \mathrm{D} 3 ; \mathrm{D} 4\}$ & 116.54 & 21.53 & -0.960 \\
\hline $\mathrm{AKM}-\{\mathrm{F} 3 ; \mathrm{D} 1 ; \mathrm{D} 2 ; \mathrm{D} 3 ; \mathrm{D} 4\}$ & 105.94 & 19.63 & -0.955 \\
\hline $\mathrm{AKM}-\{\mathrm{P} 2 ; \mathrm{P} 3 ; \mathrm{P} 4 ; \mathrm{D} 1 ; \mathrm{D} 2 ; \mathrm{D} 3 ; \mathrm{D} 4\}$ & 98.47 & 17.88 & -0.942 \\
\hline $\mathrm{AKM}-\{\mathrm{P} 2 ; \mathrm{A} 2\}$ & 135.84 & 24.66 & -0.960 \\
\hline \multicolumn{4}{|l|}{ Stage III } \\
\hline AKM & 62.50 & 5.74 & -0.994 \\
\hline $\mathrm{AKM}-\{\mathrm{P} 2 ; \mathrm{P} 3 ; \mathrm{P} 4 ; \mathrm{D} 1 ; \mathrm{D} 2 ; \mathrm{D} 3 ; \mathrm{D} 4 ; \mathrm{A} 2 ; \mathrm{A} 3 ; \mathrm{A} 4 ; \mathrm{R} 2 ; \mathrm{R} 3\}$ & 54.40 & 5.02 & -0.998 \\
\hline $\mathrm{AKM}-\{\mathrm{P} 3 ; \mathrm{P} 4 ; \mathrm{D} 1 ; \mathrm{D} 2 ; \mathrm{D} 3 ; \mathrm{D} 4 ; \mathrm{A} 2 ; \mathrm{R} 2\}$ & 51.30 & 4.29 & -0.997 \\
\hline $\mathrm{AKM}-\{\mathrm{P} 2 ; \mathrm{P} 3 ; \mathrm{P} 4 ; \mathrm{D} 1 ; \mathrm{D} 2 ; \mathrm{D} 3 ; \mathrm{D} 4 ; \mathrm{A} 2 ; \mathrm{A} 3 ; \mathrm{A} 4\}$ & 50.50 & 4.02 & -0.998 \\
\hline $\mathrm{AKM}-\{\mathrm{P} 2 ; \mathrm{P} 3 ; \mathrm{P} 4 ; \mathrm{D} 1 ; \mathrm{D} 2 ; \mathrm{D} 3 ; \mathrm{D} 4 ; \mathrm{A} 3 ; \mathrm{A} 4\}$ & 50.24 & 4.08 & -0.998 \\
\hline $\mathrm{AKM}-\{\mathrm{P} 4 ; \mathrm{D} 1 ; \mathrm{D} 2 ; \mathrm{D} 3 ; \mathrm{D} 4\}$ & 50.82 & 4.15 & -0.996 \\
\hline
\end{tabular}


For AKM- $\left\{\mathrm{F}_{3}, \mathrm{D}_{1}-\mathrm{D}_{4}\right)$, the plot of $\ln \mathrm{A}$ versus $\mathrm{E}_{\mathrm{a}}$ has the highest correlation coefficient $\left(\mathrm{E}_{\mathrm{a}}=105.94 \mathrm{~kJ} / \mathrm{mol} ; \ln \mathrm{A}=19.63 ; \mathrm{r}=0.950\right)$ and is true straight line for the second stage (FWO; $E_{a}=131.42 \pm 3.02 \mathrm{~kJ} / \mathrm{mol}$ ). Whereas for AKM- $\left\{\mathrm{P}_{2}-\mathrm{P}_{4}, \mathrm{D}_{1}-\mathrm{D}_{4}, \mathrm{~A}_{2}-\mathrm{A}_{3}, \mathrm{R}_{2}, \mathrm{R}_{3}\right\}$, the plot of $\ln \mathrm{A}$ versus $\mathrm{E}_{\mathrm{a}}$ has the highest correlation coefficient $\mathrm{E}_{\mathrm{a}}=54.4 \mathrm{~kJ} / \mathrm{mol} ; \ln \mathrm{A}=5.02$ $(\mathrm{r}=0.998)$ and is a straight line for the third stage (FWO; $\left.\mathrm{E}_{\mathrm{a}}=70.75 \pm 3.26 \mathrm{~kJ} / \mathrm{mol}\right)$.

\section{Thermodynamic parameters}

As can see from the Table 7 , the value of $\Delta \mathrm{S}^{\neq}$is positive for the first stage and negative for the second and third stages. The negative values suggest that the activated complexes have a higher degree of arrangement than the initial stage. The positive values of enthalpy and free energy of activation for this compound show that the process is connected with absorption of heat is non-spontaneous in nature ${ }^{25,26}$.

Table 7. Kinetic and thermodynamic parameters for the thermal behaviour of HBAPPA by Kissinger method

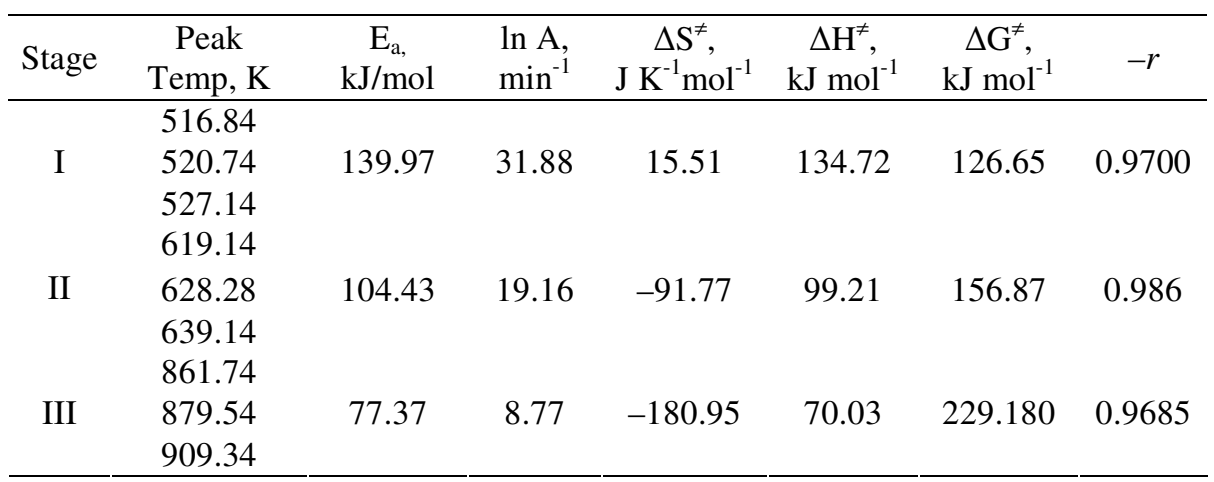

\section{Conclusion}

The title compound is decomposed in three stages. The energy of activation of first stage is more than that of the other two stages, which indicates that the first stage decompose in rather slow. $\Delta \mathrm{G}^{\neq}$is more positive indicating that the decomposition process is nonspontaneous in nature.

\section{References}

1. Flynn J H and Wall L A, J Res Natl Bur Stand A. Phys Chem., 1966, 70, 487.

2. Ozawa T, Bull Chem Soc Jpn., 1965, 38, 1881.

3. Fernandes Jr. V J and Araujo A S, Thermochim Acta., 1995, 255, 273-280.

4. Fernandes Jr. V J, Araujo A S and Fernandes G J T, J Therm Anal Calorim., 1999, 56, 811-817.

5. Andy Hor T S and Phang L T, Thermochim Acta., 1991, 178, 287-293.

6. Barral L, Cano J, Lopez J, Lonez-Bueno I, Nogueria P, Ramirez C and Abad M J, J Thermal Anal Calorim., 1999, 55, 37-45.

7. Singh H L, Sharma M, Gupta M K and Varshney A K, Bull Polish Acad Sci Chem., 1999, 47(2), 103-110.

8. Tian, Laijin, Jumlian T, Li-Tantuan, Li Guizli and Bisiwei, GaoEnquing, Liu Shuxiang, Huaxueshiji, 1999, 118, 114-116. 
9. Goldberg D E, Sharma V, Oksman A, Gluzman I Y, Wellems T E and PiwnicaWorms D, J Biol Chem., 1997, 272(10), 6567-6572.

10. Pattubala A N R, Munirathinam N and Akhil R C, Eur J Inorg Chem., 2004, 7, 1440-1446.

11. Pattubala A N R, Munirathinam N and Akhil R C, Inorg Chim Acta., 2002, 337, 450-458.

12. Valentova J, Zemlicka M, Vanickova M and Labud J, A Monograph Series of the International Conferences on Co-ordination Chemistry held Periodically at Smolenice in Slovakia, 1997, 3, 251-256.

13. Li Wrije, Yang Lin, Dongnei C, Linkunhua, Lou Quanling and Shizan, Huaxue Xuabao, 1995, 11, 296-301.

14. Dongnei C, Li Ju, Yang Lin, Lou Quanling, Shi Zan and Lin Kunhua, Chin Chem Lett., 1994, 5, 155-156.

15. Coats A W and Redfern J P, Nature, 1964, 201, 68-69.

16. Coats A W and Redfern J P, J Polym Sci Part B Polym Lett., 1965, 3, 917.

17. Kissinger H E, Anal Chem., 1957, 29, 1702-1706.

18. Akahira T and Sunose T, Res Rep Chiba Inst Technol., 1971, 16, 22-24.

19. Tang W, Liu Y, Zhang H and Wang C, Thermochim Acta, 2003, 408(1-2), 39-43.

20. Wanjun T, Yuwen L, Hen Z, Zhiyong Wand Cunxin W, J Therm Anal Calorim., 2003, 74, 309-315.

21. Kissinger H E, J Res Nat Bur Stand., 1956, 57(4), 217-221.

22. Straszko J, Olszak-Humienik M and Mozejko J, Thermochim Acta, 1997, 292, 145-150.

23. Olszak-Humienik M and Mozejko J, Thermochim Acta, 2000, 344, 73-79.

24. Lesnikovick A I and Levchik S V, J Thermal Anal., 1985, 30, 237-262.

25. Criado J M, Perez-Maqueda L A and Sanchez-Jimenez P E, J Therm Anal Calorim., 2005, 82, 671-675.

26. Sokolshii D V and Druz V A, Introduction in Theory Heterogenous Catalysis, Vyshay Shkola, Moscow, 1981 (in Russian). 


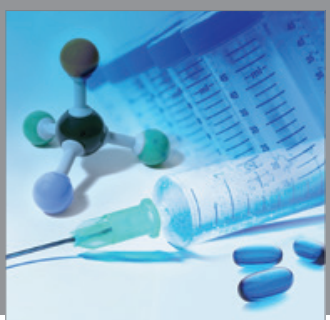

International Journal of

Medicinal Chemistry

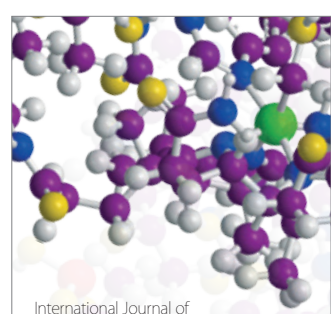

Carbohydrate Chemistry

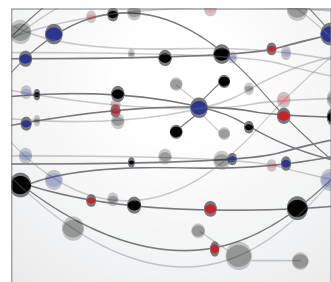

The Scientific World Journal
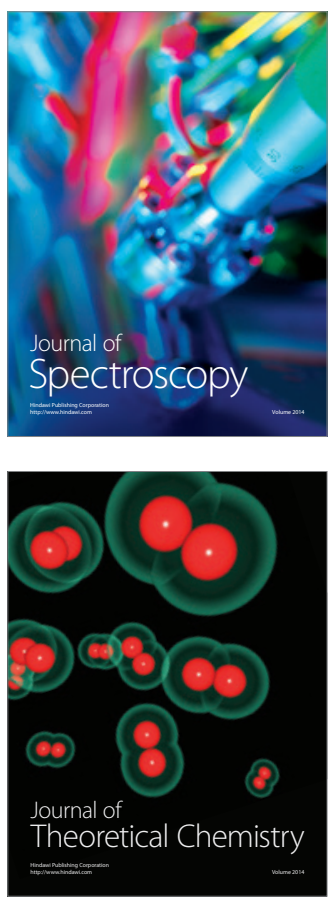
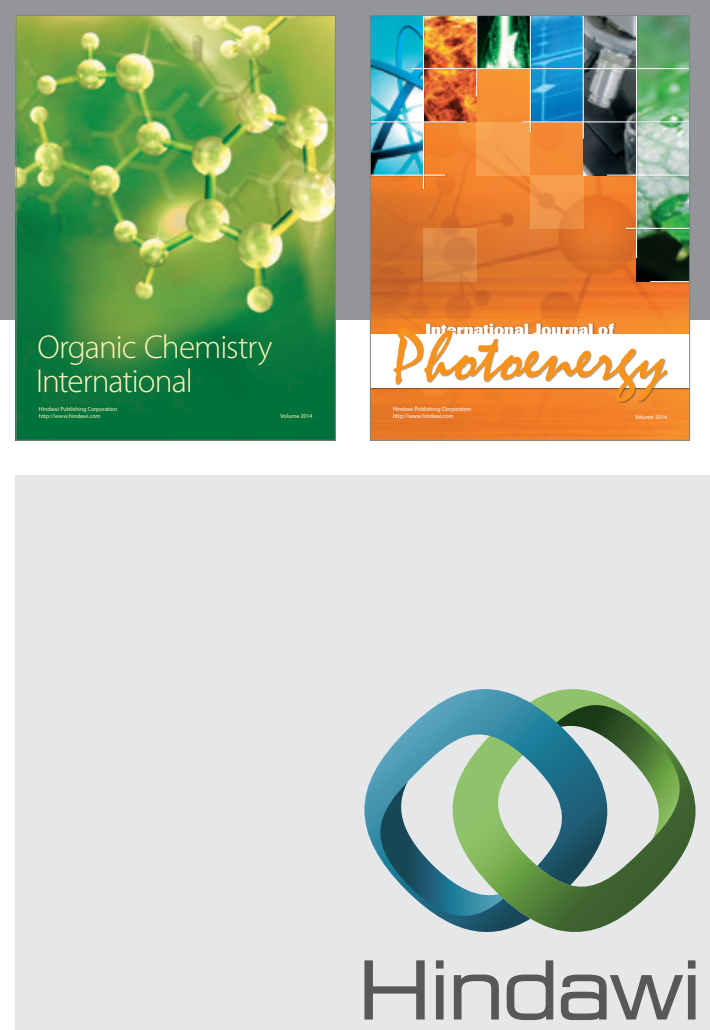

Submit your manuscripts at

http://www.hindawi.com
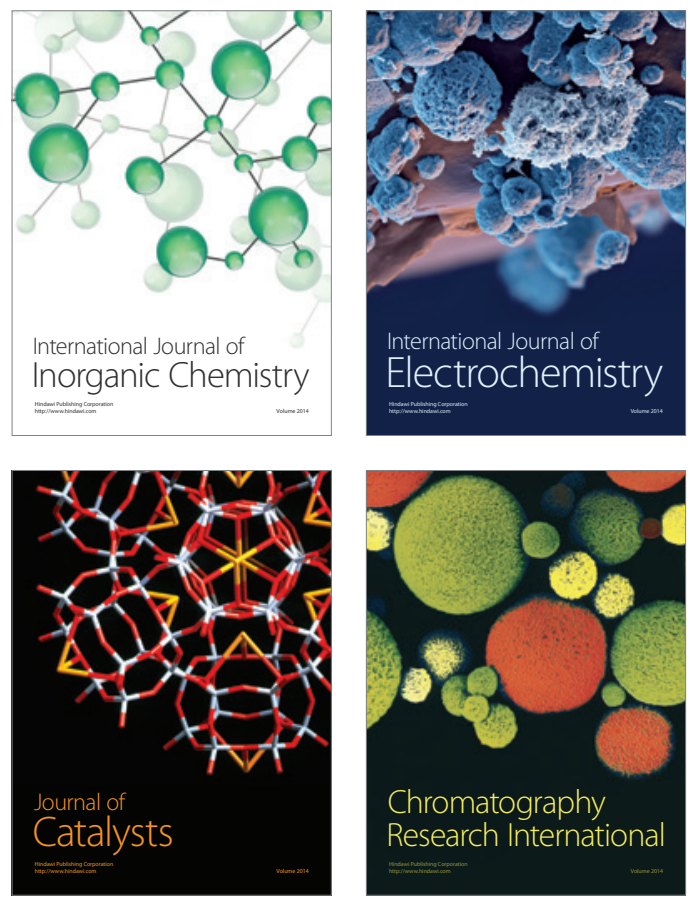
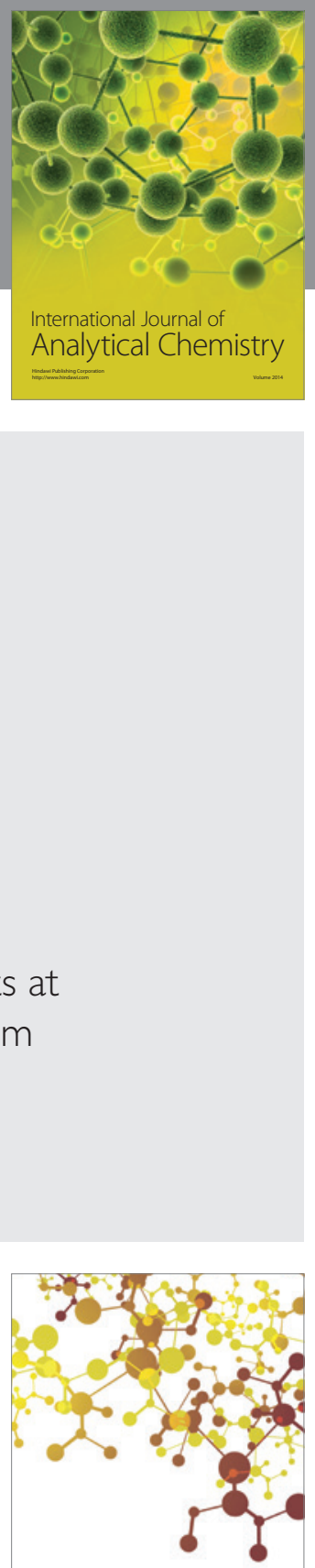

Journal of

Applied Chemistry
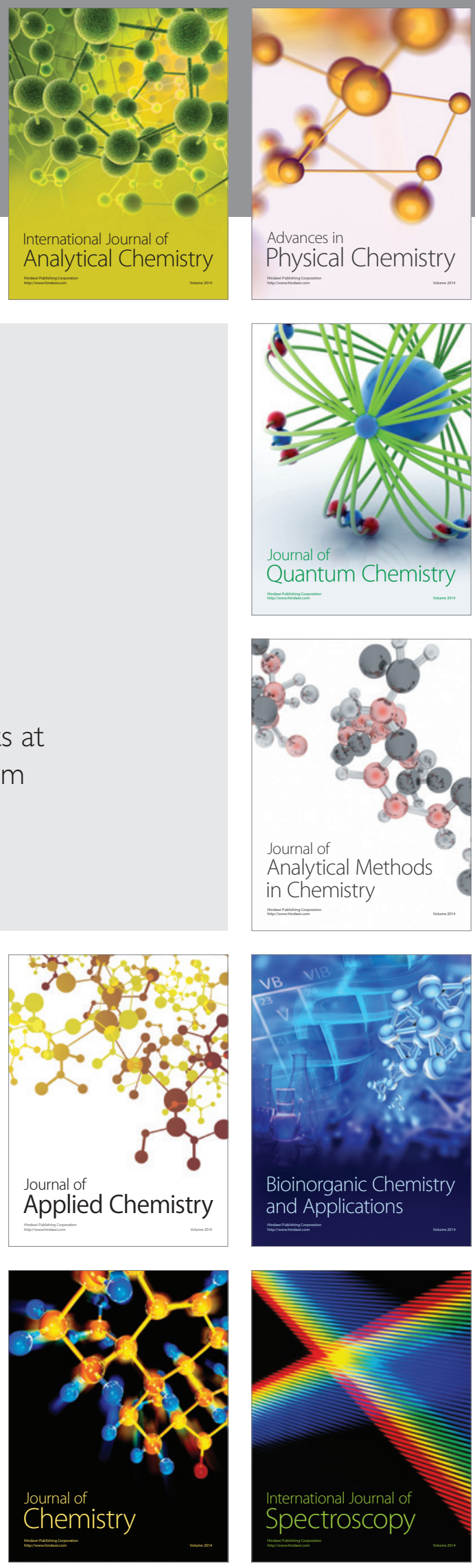